\title{
The Banach Space of Multiply Excited Atoms
}

\author{
Hubert Klar \\ University of Freiburg, Freiburg, Germany \\ Email: hubklar@aol.com
}

How to cite this paper: Klar, H. (2021) The Banach Space of Multiply Excited Atoms. Journal of Applied Mathematics and Physics, 9, 3230-3239.

https://doi.org/10.4236/jamp.2021.912211

Received: October 19, 2021

Accepted: December 28, 2021

Published: December 31, 2021

Copyright $\odot 2021$ by author(s) and Scientific Research Publishing Inc. This work is licensed under the Creative Commons Attribution International License (CC BY 4.0).

http://creativecommons.org/licenses/by/4.0/

\begin{abstract}
We present, for the first time, a unified description of adiabatic collision channels and the Wannier channel in electron-atom scattering. We identify the Wannier channel as the solution of a recently presented partial differential equation of parabolic type. The kernel of that equation has been constructed near the ionization threshold. Its eigenstates are shown to be members of a Banach space. For the purpose of demonstration, this paper embeds one adiabatic channel into a Bannach space. The full set of an adiabatic spectrum will be embedded into the Wannier continuum of a Banach space in a forthcoming paper. This technique delivers amended non-adiabatic collision channnels with ebergy-dependent potentials. That dependence manifests itself as energy-dependent discontinuity at the threshold. The branch above threshold describes the double escape of electrons, whereas the branch below threshold replaces an infinity of strongly coupled adiabatic channels by one new channel. The present paper is restricted to two-electron atoms consisting only of $s^{2}$ ${ }^{1} S$ configurations. Our model shows new unexpected effects including an electron-electron attraction similar to a Cooper pair except that our electron pair couples only to one nucleus at rest rather than to a vibrating lattice. Our electron-electron attraction stems from a dynamic deformation of the potential surface.
\end{abstract}

\section{Keywords}

Dominant Correlation, Nonseparable Wave Equations, Embedding of a Hilbert Space into a Banach Space

\section{Introduction}

Standard problems in quantum mechanics confirm that normalizable eigenstates of a Hermitian operator span a Hilbert space. This is doubtless true for one-dimensional systems described by an ordinary differential equation, and for separable more-dimensional systems. The present paper shows that a Hilbert space over the set of real numbers $\mathbb{R}$ is too small for multiplying excited atoms. 
Therefore we need an extension to a vector space over the set of complex numbers $\mathbb{C}$, i.e. the norm of an element is no longer real positive. Such vector spaces are usually referred to as Banach spaces.

A powerful strategy to solve the stationary wave equation for a many-electron atom is the employment of hyperspherical coordinates [1]. Also in these coordinates, the wave equation is non-separable, but an accurate method to calculate multiply excited energy levels has been presented long ago by Macek [2], Klar et al. [3]. To this end, we use a two-step method as follows. First, we solve the problem at constant values of the hyperradius

$$
R=\sqrt{\sum_{i} r_{i}^{2}}
$$

We apply this amended BO method to a simplified two-electron atom. That step leads to collision channels and static potentials which deliver resonance levels as matrix eigenvalues. Thus the second step requires the calculation of eigenvalues in a static potential. We did that for two-electron atoms like $\mathrm{He}$ and $\mathrm{H}^{-}$but the generalization to more electrons is obvious. Klar et al. [3] did such calculations making advantage of technical details. Both groups obtained surprisingly good results except for very highly excited channels close to the ionization limit. Thus we got the suspicion that Macek's two-step method which is basically a Born-Oppenheimer (below shortly BO) treatment is too simple. Klar [4] presented recently an amended BO-method which removes all first-order channel coupling. That method describes in the molecular case electronic eigenstates in a moving nuclear frame where fictitious forces may occur. We apply this here for the purpose of demonstrating the amended BO method to a simplified two-electron atom. To this end, we disregard any angular correlation but we treat properly radial correlation. Clearly, we are now confronted to treat in the first step an evolution equation rather than a simple linear matrix eigenvalue equation. This novel channel equation resembles the well-studied heat equation. In our problem, its fundamental solution folded which an initial state function delivers eigen-evolutions but not yet the desired improved potentials. The present paper derives exactly the evolution near zero-energy and calculates exact eigenstates. These eigen-evolutions manifest themselves as members of a Banach space that replaces a familiar Hilbert space.

The present article embeds, therefore, the Hilbert space of static BO-channels into a new Banach space spanned by the evolution continuum. Many years ago Fano [5] presented a prototype embedding of one Hilbert space into another one. Basically, we adopt Fano's procedure with necessary modifications. To our knowledge, the present way to attack the problem of resonance formation is entirely new since we present here, for the first time, a unified description of Feshbach and Wannier resonances.

This article is organized as follows. In Section 2 we summarize the use of hyperspherical coordinates in atomic structure calculations. Section 3 puts the focus on the Wannier phenomenon and derives an exact fundamental solution (also referred to as kernel). The embedding procedure of one BO collision 
channel into the Wannier continuum is the subject of Section 4. Section 5, finally, summarizes the present development and discusses aspects for future work.

\section{Hyperspherical Coordinates}

We consider a not too heavy atom with $N$ electrons, and denote their body-fixed positions with $\vec{r}_{i}, i=1, \cdots, N$ and parametrize these vectors by

$$
\vec{r}_{i}=R \hat{n}_{i}(\omega)
$$

where $R$ is the hyperradius given by (1), and $\hat{n}_{i}$ are unit vectors on the surface of the unit sphere $\mathbb{S}_{3 N-4}$. $\omega$ stands for a set of $3 N-4$ spherical angles, see for instance [1]. We exclude here any overall rotation because that has nothing to do with our principle aim. The kinetic energy of all electrons in the nucleus frame reads then

$$
T=-\frac{1}{2} R^{-(3 N-1)} \frac{\partial}{\partial R} R^{3 N-1} \frac{\partial}{\partial R}-\frac{\Lambda^{2}}{2 R^{2}}
$$

where $\Lambda^{2}$ is the generator of rotations in the space $\mathbb{R}_{3 N-4}$ [1], also refered to as grand angular momentum.

The electrostatic potential for light atoms reads

$$
V=-\sum_{i} \frac{Z}{r_{i}}+\sum_{i<j} \frac{1}{r_{i j}}
$$

with the nuclear charge $Z$ and $r_{i j}$ being the electron-electron separations. In hypershericals, the function $V$ gets the simple pleasent structure

$$
V=\frac{C(\omega)}{R}
$$

where $C(\omega)$ depends only on a set $\omega$ of $3 N-4$ angles .

The stationary wave equation has then the form

$$
\left\{-\frac{1}{2} \frac{\partial^{2}}{\partial R^{2}}-\frac{\Lambda^{2}-\lambda(\lambda+1)}{2 R^{2}}+\frac{C(\omega)}{R}-E\right\}\left(R^{(3 N-1) / 2} \Psi\right)=0
$$

with

$$
\lambda=\frac{3(N-1)}{2}
$$

Equation (6) is, of course, still nonseparable.

In order to solve it, Macek [2] starts from a channel expansion

$$
\left(R^{(3 N-1) / 2} \Psi\right)=\sum_{\mu} F_{\mu}(R) E_{\mu}(\omega ; R)
$$

We [4] replace now in the channel equation the first derivative of the radial function $F(R)$ by its logarithmic one and use the Sommerfeld approximation for the logarithmic derivative ${ }^{1}$

$$
\frac{F^{\prime}}{F}=i K(R)
$$

${ }^{1}$ Sommerfeld used that Abstrahlungsbedingung for light; we do it here for electrons. 
to get an improved channel equation

$$
\left\{-i K \frac{\partial}{\partial R}+\frac{\Lambda^{2}}{2 R^{2}}+\frac{C(\omega)}{R}\right\} E_{\mu}=V(R) E_{\mu}
$$

The desired separation is now, except for the second-order coupling term $\frac{\partial^{2} E_{\mu}}{\partial R^{2}}$ complete because Equation (9) depends no longer on the unknown radial function. That 2 nd order coupling is not relevant for us because it operates in the inner reaction zone rather than in the Coulomb zone.

To this end, we arrive at the recently presented eigen-evolution equation [6]

$$
i K \frac{\partial E_{\mu}}{\partial R}=\left(\frac{\Lambda^{2}}{2 R^{2}}+\frac{C(\omega)}{R}-V(R)\right) E_{\mu}
$$

Equation (11) resembles the heat equation. Actually, the lhs of (11) may be regarded as a time-derivative provided we treat the hyperradius as classical coordinate as function of time, say $R(t)$. The momentum $K$ is then $K=\dot{R}$, i.e.

$K \frac{\partial}{\partial R}=\frac{\partial}{\partial t}$. But it is here not necessary to employ classical mechanics.

The above development merits one more comment. Equation (11) is an extension of the familiar BO approximation. Disregarding the derivative on the lhs of (11) we fall back to the standard eigenvalue problem of BO. Our (11), however, describes an evolution along the coordinate $R$. Therefore we work here with angular modes on a breathing sphere in contrast to all previous works which work on a static sphere. In the molecular case, we would arrive at electronic functions on a moving nuclear frame. Here we treat angular functions on the surface of a breathing sphere.

The next Section 3 exactly calculates the evolution departing from any initial two-electron state.

\section{The Wannier Mode as Evolution}

We start now looking into the potential surface of a two-electron atom-like He. This shows one saddle point. But only its unstable component describing radial correlation leads to an unusual ionization cross-section [7] and is expected to cause surprises also below threshold in agreement with our previous studies [6]. In order to explore radial correlation effects, we freeze below the angular correlation and study the unstable radial correlation alone. That restriction eliminates also the 1 -degeneracy which has nothing to do with the Wannier phenomenon.

Hypesperical coordinates are very suitable for that because they have an important property. Let us consider a Shere $\mathbb{S}_{n}$. That can be mapped with $n$ polar angles. $n-1$ of them are meridian angles on intervals $[0, \pi]$ and one azimuth angle running on the hyperspherical equator with range $[0,2 \pi]$. We denote that angle with $\varphi \in[0,2 \pi]$. According to Sommerfeld [6] $\varphi$ is in our situation given by

$$
\varphi=\tan ^{-1} \frac{r_{1}^{2}-r_{2}^{2}}{2 r_{1} r_{2}}
$$


The charge function reads then

$$
C(\varphi)=-Z\left(\frac{1}{\sqrt{1-\sin \varphi}}+\frac{1}{\sqrt{1+\sin \varphi}}\right)+\frac{1}{\sqrt{1-\cos \varphi}}
$$

That function has a smooth maximum located at $\varphi=\pi$, and may be approximated there by

$$
C(\varphi)=-C_{0}-C_{2}(\varphi-\pi)^{2}
$$

with the coefficients [3]

$$
C_{0}=\frac{4 Z-1}{\sqrt{2}}
$$

and

$$
C_{2}=\frac{12 Z-1}{4 \sqrt{2}}
$$

We come now to the evolution Equation (10). The wavenumber $K(R)$ is related to the conservation of energy on the top of the potential ridge by

$$
E=\frac{1}{2} K^{2}-\frac{C_{0}}{R}
$$

At threshold for double escape $E=0$ the electron pair is in equilibrium on the top of the ridge where the kinetic radial energy compensates their potential energy. From (17) we get immediately

$$
K=\sqrt{\frac{2 C_{0}}{R}}
$$

see also Wannier [7]. For high energy, we would find from (17) of course $K=\sqrt{2 E}$ whereas in the discrete target spectrum we find $K=i \frac{Z}{n}$. Moreover, the Sommerfeld angle $\varphi$ is a cyclic coordinate in the Laplacian. That simplifies the further investigation. Actually, our centrifugal term reads simply

$$
\Lambda^{2}=-4 \frac{\partial^{2}}{\partial \varphi^{2}}
$$

see [3] with putting there $\psi=0$.

Thus we arrive at the wave equation for the evolution

$$
i \sqrt{\frac{2 C_{0}}{R}} \frac{\partial E}{\partial R}+\frac{2}{R^{2}} \frac{\partial^{2} E}{\partial \varphi^{2}}+\frac{C_{0}}{R} E+\frac{C_{2}}{R}(\varphi-\pi)^{2} E=W E
$$

In order to solve it, we put

$$
E(R, \varphi)=\exp \{i \sqrt{R} D(\varphi)\}
$$

and get for the function $D(\varphi)$ the relation

$$
-\sqrt{\frac{C_{0}}{2}} \frac{D}{R}-\frac{2}{R}\left(D^{\prime}\right)^{2}-2 i R^{-3 / 2} D^{\prime \prime}+\frac{C_{0}}{R}+\frac{C_{2}}{R}(\varphi-\pi)^{2}=W(R)
$$

That we solve with the Ansatz 


$$
D(\varphi)=\kappa+\lambda(\varphi-\pi)^{2}
$$

and find for the coefficients

$$
\kappa=\sqrt{2 C_{0}}
$$

and for $\lambda$ we get from

$$
\lambda^{2}+\frac{1}{8} \sqrt{\frac{C_{0}}{2}} \lambda-\frac{C_{2}}{8}=0
$$

two real solutions are given by

$$
\begin{aligned}
& \lambda_{1}=\frac{1}{16}\left(\sqrt{\frac{C_{0}+32 C_{2}}{2}}+\sqrt{\frac{C_{0}}{2}}\right)>0 \\
& \lambda_{2}=\frac{1}{16}\left(-\sqrt{\frac{C_{0}+43 C_{2}}{2}}+\sqrt{\frac{C_{0}}{2}}\right)<0
\end{aligned}
$$

The $\lambda$ in (25) describes a potential surface deformation. Surprisingly that is different for outgoing waves $\left(\lambda_{1}\right)$ and incoming waves $\left(\lambda_{2}\right)$, respectively.

The eigenvalues are then given by

$$
W(R)=\frac{4 \lambda}{R^{3 / 2}}(-i)
$$

Imaginary eigenvalues (28) are not acceptable for potential energy. An eigenvalue of a Hermitian operator is manifestly real.

An earlier treatment of the present problem based on matrix calculus showed the same difficulty except for the ground channel which carries only singly excited bound states [8].

The evolution folded with an asymptotic state is certainly a member of a vector space with scalar product and a norm. We conclude from (28) that we are confronted here with a norm over the field of complex numbers $\mathbb{C}$. This is in contrast to a familiar Hilbert space whose norm runs over positive real numbers $\mathbb{R}$. The evolutions constitute therefore elements of a Banach space. That result is rather unusual in atomic structure theory since textbooks generally teach that the eigenstates of physically meaningful operators span a Hilbert space. That standard statement is in fact correct as long as the wave equation is either one-dimensional or it is a separable partial differential equation. Our situation treats however a non-separable partial differential equation of parabolic type.

\section{Embedding of one Hilbert Space Element into a Banach Space}

Embeddings are nontrivial procedures. Although Fano [5] embedded one Hilbert space into another larger Hilbert space we are allowed to adopt his general procedure to go ahead with substantial changes necessary for our problem, however. For the purpose of illustration, we embed in this paper only one Hilbert space element into a Banach space.

Below we employ a more compact description of the basic equations. To this 
end, we identify an improved electron-atom/ion potential as an eigenvalue of the reduced Hamiltonian

$$
h=h_{0}+h_{1}
$$

where $h_{0}$ is the adiabatic Hamiltonian, and $h_{1}$ is the non-adiabatic coupling given by

$$
h_{1}=-i K(R) \frac{\partial}{\partial R}
$$

$h_{0}$ creates a descrete spectrum of channel potentials, whereas $h_{1}$ opens a continuum [10].

The present paper studies the simplest case of only one descrete channel identified by its channel wavefunction $\Phi$ and its potential eigenvalue $U(R)$, i.e. we write

$$
\langle\Phi|h| \Phi\rangle=U
$$

The evolution folded with any initial value function leading to a real potential $W$ we denote that state by $\Gamma(W)$, and rewrite (28) in the form

$$
\frac{\left\langle\Gamma(W)|h| \Gamma\left(W^{\prime}\right)\right\rangle}{\left\langle\Gamma(W) \mid \Gamma\left(W^{\prime}\right)\right\rangle}=W(R)=\frac{4 \lambda}{R^{3 / 2}}
$$

with the necessary Banach norm

$$
\left(\Gamma(W) \mid \Gamma\left(W^{\prime}\right)\right)=-i \frac{\sqrt{R}}{\pi} \delta\left(W-W^{\prime}\right)
$$

We have already seen that the modified ridge curvature $\lambda$ has two eigenvalues, $\lambda_{1}>0$ and $\lambda_{2}<0$. Therefore we treat separately the functions $\Gamma(W>0)=\Gamma_{1}(W)$, and $\Gamma(W<0)=\Gamma_{2}(W)$. We will see shortly that $W>0$ describes expanding three-body complexes whereas $W<0$ delivers shrinking complexes. Thus we modify (32) to

$$
\left\langle\Gamma_{i}(W)|h| \Gamma_{j}\left(W^{\prime}\right)\right\rangle=W_{i}(R)\left\langle\Gamma_{i}(W) \mid \Gamma_{j}\left(W^{\prime}\right)\right\rangle \delta_{i j}
$$

$i, j=1,2$, and with the norm analogous to (33).

With these ingredients, we attack now the eigenvalue equation

$$
h \Psi=V(R) \Psi
$$

To this end, we diagonalize $h$ in the basis given by $\Phi$ and the two $\Gamma_{j}, j=1,2$. Therefore we express the solution $\Psi(R)$ as a linear combination [5] [9]

$$
\Psi=A \Phi+\int_{0}^{\infty} B\left(W, W^{\prime}\right) \Gamma_{1}\left(W^{\prime}\right) \mathrm{d} W^{\prime}+\int_{-\infty}^{0} B\left(W, W^{\prime}\right) \Gamma_{2}\left(W^{\prime}\right) \mathrm{d} W^{\prime}
$$

We stress there is only one single function $B\left(W, W^{\prime}\right)$, but it enters in (36) on different energy segments.

Now we perform the diagonalization, and multiply (35) from left with $\Gamma_{j}\left(W^{\prime}\right)$ and integrate over the ridge angle $\varphi$. This step leads to

$$
\left(V-W^{\prime}\right) B\left(W, W^{\prime}\right)=i X_{j}\left(W^{\prime}\right)
$$

$j=1,2$. From (36) we get the solutions 


$$
B\left(W, W^{\prime}\right)=i \mathfrak{P} \frac{X_{1}\left(W^{\prime}\right)}{V-W^{\prime}}
$$

for $W>0$, and

$$
B\left(W, W^{\prime}\right)=i \mathfrak{P} \frac{X_{2}\left(W^{\prime}\right)}{V-W^{\prime}}
$$

for $W<0$, respectively. The symbol $\mathfrak{P}$ stands for the Cauchy principal value and $X_{j}$ is given by the coupling

$$
X_{j}=\left(\Gamma_{j}(W)|h| \Phi\right)
$$

Now we multiply (35) with $\Phi$ and integrate again over $\varphi$, and now we substitute the above values for $B\left(W, W^{\prime}\right)$. With the coupling

$$
Y_{j}(W)=\left(\Phi|h| \Gamma_{j}(W)\right)
$$

we arrive at improved potentials

$$
V_{1}=U+i \mathfrak{P} \int_{0}^{\infty} \frac{X_{1}\left(W^{\prime}\right) Y_{1}\left(W^{\prime}\right)}{V_{1}-W^{\prime}}
$$

above the ionization threshold, and

$$
V_{2}=U-i \mathfrak{P} \int_{-\infty}^{0} \frac{X_{3}\left(W^{\prime}\right) Y_{2}\left(W^{\prime}\right)}{V_{2}-W^{\prime}}
$$

below threshold. In (40), (41) the principle value integrals represent a shift of the adiabatic potential due to the coupling to the Wannier continuum. That shift is real as may be sen as follows. In order to return to observable quantities we must devide all potential contribution by the Banach normalization given by $-i \frac{\sqrt{R}}{\pi}$. That cancels the factor $i$ in front of the integrals in Eqns. (41) and (42).

Remarkably we get above and below threshold different potentials. But this is not unexpected because 1) the potential surface deformation depends on the direction of flow, and 2) because any description beyond an adiabatic treatment should be energy-dependent. Actually our $V_{1}$ and $V_{2}$ describe a shift above/ below threshold. An alternative statement reads that the improved potential has a discontinuity at threshold $(E=0)$.

\section{Summary and Conclusions}

This paper has presented a new collision channel equation beyond a static BO approximation. A partial differential equation of parabolic type has been used to treat inelastic electron-atom scattering near an ionization threshold. The solution of that equation has been identified as Fresnel distribution [10]. This distribution folded with any atomic asymptotic state delivers collision channels for electron scattering from excited atoms. In contrast to standard treatments the Fresnel-eigenstates span a Banach space rather than a Hilbert space. That larger space contains besides the standard Feshbach inelastic channels of the reaction $e+A\left(E_{n}\right)^{*}$, also the Wannier channel present near ionization thresholds. Embedding of the Hilbert space generated by ordinary Feshbach channels into the nov- 
el Banach space leads, for the first time, to a unified description of all collision channels of one system. The embedding leads to improved electron-atom* potentials beyond static models. The potential of the Wannier channel is as expected energy-dependent. That potential energy dependence manifests itself as discontinuity at threshold. Below threshold the Wannier channel delivers a potential given by

$$
W=\frac{4 \pi \lambda_{2}}{R^{2}}<0
$$

where $R$ is the hyperradius of both excited electrons, and above threshold

$$
W=\frac{4 \pi \lambda_{1}}{R^{2}}>0
$$

where the $\lambda_{i}$ are generalized potential surface curvatures for shrinking and expanding modes of the excited complex $A^{* *}$. Equations (43) and (44) represent unusual centrifugal describing the electrostatic potential of a dominantly correlated electron pair in the field of a nucleus. Above threshold, our Wannier channel delivers the Wannier ionization threshold law [7].

The problem treated in this paper was a hot topic for decades. But a convincing solution to it was not found. Under these circumstances, the community has lost interest in further investigations. The present paper has presented an outstanding solution. The experimentalists are invited to perform highly accurate measurements slightly below the ionization threshold (not more than $3 \mathrm{eV}$ below) to search for narrow resonances. Also, theoreticians are invited to do new computational work.

In summary, this paper has opened an entirely new field of atomic structure theory. Standard Feshbach resonances and the new Wannier resonances are treated in one single Banach space.

\section{Acknowledgements}

The author thanks his former teacher Professor Ugo Fano for encouraging him to attack the present problem. The author is grateful for much financial support from the DFG.

\section{Conflicts of Interest}

The authors declare no conflicts of interest regarding the publication of this paper.

\section{References}

[1] Morse, P.M. and Feshbach, H. (1953) Methods of Mathematical Physics. Dover Publishing, Dover, NY.

[2] Macek, J.H. (1968) Study of Doubly Excited States of Two-Electron Atoms. Journal of Physics B, 1, 811. https://doi.org/10.1088/0022-3700/1/5/309

[3] Klar, H. and Klar, M. (1980) Accurate Calculationof Doubly Excited States of Two-Electron Atoms Using Hypersherical Coordinates. Journal of Physics B: Atomic 
and Molecular Physics, 13, Article No. 1057. https://doi.org/10.1088/0022-3700/13/6/014

[4] Klar, H. (2020) The Born-Oppenheimer Approximation Revisited. Journal of Applied Mathematics and Physics, 8, 1507-1514. https://doi.org/10.4236/jamp.2020.88116

[5] Fano, U. (1961) Configuration Interaction Effects on Intensities and Phaseshifts. Physical Review Journals Archive, 124, Article No. 1866. https://doi.org/10.1103/PhysRev.124.1866

[6] Sommerfeld, A. (1944) Theory of Atomic Spectra. Springer, Berlin.

[7] Wannier, G. (1953) Threshold Ionization of Hydrogen Atoms by Electons. Physical Review Journals Archive, 90, Article No. 817. https://doi.org/10.1103/PhysRev.90.817

[8] Klar, H. (2020) Theory of the Wannier Phenomenon. Journal of Applied Mathematics and Physics, 8, 2416-2426. https://doi.org/10.4236/jamp.2020.811178

[9] Klar, H. and Fano, U. (1976) Postadiabatic Analysis of Atomic Collisions. Physical Review Letters, 37, Article No. 1132. https://doi.org/10.1103/PhysRevLett.37.1132

[10] Walter, W. (1994) Einführung in die Theorie der Distribtionen. Bibliographic Institute, Mannheim. 